\title{
DEFINICIÓN ACTUAL Y TRATAMIENTO DE LA EYACULACIÓN PRECOZ
}

\author{
Walter Cardona Maya.
}

Grupo Reproducción. Facultad de Medicina. Universidad de Antioquia. Medellín. Colombia.

Resumen.- OBJETIVO: Discutir el concepto sobre la definición actual y el tratamiento de la eyaculación pre$\mathrm{COZ}$.

MÉTODOS: Se realizó una evaluación detallada de la actual definición y tratamiento de la eyaculación preCOZ.

RESULTADOS: Se encontraron varias definiciones, sin embargo no hay un consenso sobre cómo definir la eyaculación precoz. Adicionalmente, existen varios tratamientos: diario, sobre demanda y agentes tópicos.

CONCLUSIONES: La eyaculación precoz implica tres características específicas: corto tiempo de latencia eyaculatoria intravaginal, falta de control, e insatisfacción sexual.

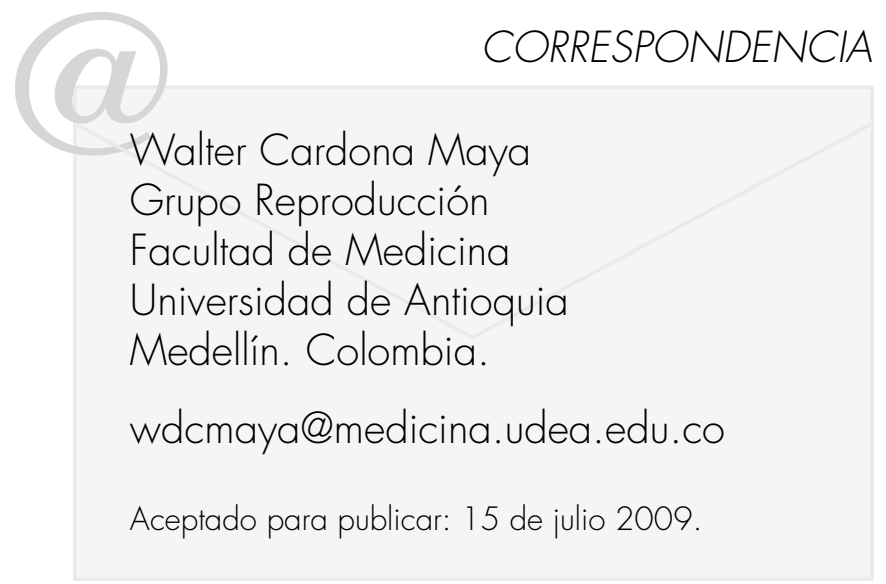

Palabras clave: Eyaculación precoz. Tratamiento. Fertilidad. Sexualidad.

Summary.- OBJECTIVES: To discuss a concept about the current definition and treatment of premature ejaculation.

METHODS: A detailed review was performed on the current definition and treatment of premature ejaculation.

RESULTS: Several definitions were found, however a consensus on how to define premature ejaculation is lacking. In addition, there are several treatments: daily, on demand and topical agents.

CONCLUSIONS: Premature ejaculation implicated three specific characteristics: short intravaginal ejaculatory latency time, lack of control, and sexual dissatisfaction.

Keywords: Premature ejaculation. Treatment. Fertility. Sexuality.

\section{INTRODUCCIÓN}

La eyaculación es un proceso mediado principalmente por el sistema nervioso autónomo y consta de dos fases principales: la emisión y la expulsión del semen (1). El eyaculado varía considerablemente respecto al volumen entre individuos, pero usualmente es entre 2 y $6 \mathrm{~mL}$ en volumen, $>2 \mathrm{~mL}$ acorde a los valores de referencia de la OMS de 1999 (2). Las vesículas seminales producen alrededor de $40-80 \%$, la próstata contribuye con alrededor de 10-30\%, el bulbo uretral y las glándulas uretrales también secretan una pequeña cantidad de alrededor de $2-5 \%$ del volumen total del eyaculado. 


\section{Eyaculación precoz}

Durante la evolución de la sexualidad humana, la habilidad para controlar el tiempo de eyaculación ha sido una de las más importante características de la salud sexual de las parejas. Por esta razón, la carencia de control en la eyaculación tiene un profundo efecto psicológico de base y su tratamiento debe ser realizado mediante una terapia sicológica al hombre o a la pareja. Las alteraciones eyaculatorias, especialmente la eyaculación precoz (EP) es uno de las mas comunes complicaciones de los hombres adultos (3-4) y hombres con EP reportan bajos niveles de satisfacción sexual comparado con hombres con una normal eyaculación.

$E P$, o falta de control de la eyaculación, es la incapacidad de ejercer un control voluntario sobre el reflejo eyaculatoria por lo que una vez que un hombre llega a un cierto nivel de excitación sexual eyacula automática y rápidamente después o incluso antes de la penetración vaginal. Debido a que la eyaculación intravaginal en pacientes con EP usualmente ocurre, rara vez la EP es causa de infertilidad. Si llegado el caso, la eyaculación sucede antes de la penetración vaginal, las parejas pueden realizar algún procedimiento de reproducción asistida para solucionar el problema de infertilidad.

No hay una definición universalmente aceptada para la eyaculación precoz debido básicamente a la carencia de instrumentos que permitan la detección específica de esta alteración. Por lo tanto, no existe un consenso de cómo se define la EP, y varias definiciones existen, por ejemplo:

1) Asociación Americana de Urología: "Eyaculación que ocurren antes de lo deseado, ya sea antes o poco después de la penetración, causando angustia a uno o a ambos compañeros" (5).

\section{2) Consultor Internacional sobre Disfunción Eréctil:} "eyaculación con mínima estimulación antes de lo deseado y antes o poco después de la penetración, lo que causa molestia o angustia, con poco o ningún control voluntario por parte del paciente" (6-7).

\section{3) Manual Diagnostico y Estadístico de la Sociedad Americana de Psiquiatría 4ta edición (DSM-IV-TR):} "Eyaculación persistente o recurrente con un mínimo de estimulación antes o poco después de la penetración, y antes que el individuos lo desee" (6-7).

4) Organización Mundial de la Salud: "una incapacidad para retrasar la eyaculación lo suficiencia para disfrutar del acto sexual, se manifiesta con una de las siguientes características: a) aparición de la eya- culación antes o poco después del inicio del coito (si el plazo es necesario: antes o dentro de los $15 \mathrm{se}$ gundos del comienzo del coito); y b) la eyaculación se produce en ausencia de erección suficiente para hacer posible el coito". Además, la definición de la OMS excluye a los hombres cuya EP es debida al alcohol, el abuso de sustancias o medicamentos, y la baja frecuencia de la actividad sexual

5) Sociedad Internacional de Medicina Sexual: durante el 2007, respondiendo a la variabilidad de definiciones en todo el mundo sobre y la necesidad por un estándar universal, la Sociedad Internacional de Medicina Sexual estableció un ad hoc comité constituido por 21 reconocidos expertos para establecer la nueva definición de EP, y estos expertos clínicos y básicos concluyeron que "la EP es una disfunción sexual caracterizada por una eyaculación en la cual siempre o casi siempre se produce antes o dentro de aproximadamente un minuto después de la penetración vaginal, y la incapacidad para retrasar la eyaculación en todas o casi todas las penetración vaginales; y consecuencias personal negativas como angustia, molestia, frustración y la negativa de tener intimidad sexual" (8).

En resumen, la EP complica tres específicas características: corto tiempo de latencia eyaculatoria intravaginal (TLEI), falta de control e insatisfacción sexual.

\section{Tratamiento de la Eyaculación Precoz}

El tratamiento de la PE abarcado intervenciones psicológicas, comportamentales y farmacológicas:

a) Inhibidor selectivo de la recaptación de serotonina (ISRS): Aumenta el nivel extracelular del neurotransmisor serotonina mediante la inhibición de su recaptación dentro de la celula presináptica, aumentando el nivel de serotonina disponible para unirse a los receptores postsinápticos. ISRS abarcan 5 compuestos: paroxetina, fluoxetina, sertralina, citolopram y fluoxamina. El tratamiento diario con $20-40 \mathrm{mg}$ de paroxetina, aumenta el TLEI alrededor de 8,8 veces en comparación con el placebo (9).

b) Clomipramina: Un antidepresivo tricíclico, es un fuerte, pero no completamente ISRS, como el principal metabolito activo desmetilclomipramina actúa preferentemente como un inhibidor de recaptación de norepinefrina. La administración de clomipramina, 4 a 6 horas antes del coito es eficaz y bien tolerado, pero se asocia con menos demora eyaculatoria que el tratamiento diario. Es importante mencionar que la 
administración diaria de un ISRS se asocia con mas altos TLEI en comparación con la administración de varias horas antes de la relación sexual (9).

d) Dapoxetina: un ISRS de acción corta que se encuentra actualmente en fase de desarrollo para el tratamiento de EP, tiene un perfil farmacocinética que permite un progreso relativamente rápido de las concentraciones séricas y una rápida eliminación después de la dosificación oral, que puedan contribuir a su utilidad como terapia en la demanda de EP. HeIlstrom WJ, et al., Demostró que dapoxetina administrada 1 a 2 horas antes del tiempo planead para la relación sexual aumentó el TLEI en comparación con el placebo en casi 1 minuto (10).

e) Tramadol: Un analgésico opioide sintético que está disponible en forma genérica en la mayoría de los países, puede ser eficaz para el tratamiento sobre demanda de la EP. Safarinejad MR et al., (1 1) y Salem $E A$ et al., (12) demostraron en pacientes con $P E$, que sobre demanda, 2 horas antes de la relación sexual, aumenta el TLEI.

f) Agentes tópicos: anestésicos tópicos locales como las formulaciones de lidocaína o prilocaína en crema, gel o aerosol muestran de manera eficaz la desensibilización, y han demostrado que aumentan la media de TLEl. Además, un agente tópico de Asia se aplica al glande $1 \mathrm{~h}$ antes de la relación sexual y se ha demostrado en un estudio, doble ciego, que retrasa la eyaculación y mejora la satisfacción sexual. Sin embargo, se observan efectos secundarios como dolores locales y quemaduras leves (13-14).

\section{BIBLIOGRAFÍA y LECTURAS RECOMENDADAS ( ${ }^{*}$ lectura de interés $y^{* *}$ lectura fundamental)}

1. Levitas E, Lunenfeld E, Weiss N, Friger M, HarVardi I, Koifman A, et al. Relationship between the duration of sexual abstinence and semen quality: analysis of 9,489 semen samples. Fertil Steril, 2005; 83: 1680.

2. WHO. WHO Laboratory Manual for the Examination of Human Semen and Sperm-Cervical Mucus Interaction. Cambridge: Cambridge University Press, 1999.
3. Vanden Broucke H, Everaert K, Peersman W, Claes H, Vanderschueren D, Van Kampen M. Ejaculation latency times and their relationship to penile sensitivity in men with normal sexual function. J Urol, 2007; 177, 237.

4. Wolters JP, Hellstrom WJ. Current concepts in ejaculatory dysfunction. Rev Urol, 2006; 4: S18.

5. Montague DK, Jarow J, Broderick GA, Dmochowski RR, Heaton JP, Lue TF, et al. AUA guideline on the pharmacologic management of premature ejaculation. J Urol, 2004; 172: 290.

**6. Broderick GA. Premature ejaculation: on defining and quantifying a common male sexual dysfunction. J Sex Med, 2006; 3: 295.

*7. Lue TF, Giuliano F, Montorsi F, Rosen RC, Andersson KE, Althof S, et al. Summary of the recommendations on sexual dysfunctions in men. $\mathrm{J}$ Sex Med, 2004; 1: 6.

*8. ISSM. ISSM announces new definition of premature ejaculation. International Society of Sexual Medicine Newsbulletin, 2007; 24: 6.

9. Waldinger MD, Berendsen HH, Blok BF, Olivier B, Holstege G. Premature ejaculation and serotonergic antidepressants-induced delayed ejaculation: the involvement of the serotonergic system. Behav Brain Res, 1998; 92: 111.

10. Hellstrom WJ, Gittelman M, Althof S. Dapoxetine $\mathrm{HCl}$ for the treatment of premature ejaculation: $\mathrm{A}$ Phase II, randomised, double-blind, placebo controlled study. J Sex Med., 1: 59:[abstract \# O97], 2004.

11. Safarinejad MR, Hosseini SY. Safety and efficacy of tramadol in the treatment of premature ejaculation: a double-blind, placebo-controlled, fixeddose, randomized study. J Clin Psychopharmacol, 2006; 26:27.

12. Salem EA, Wilson SK, Bissada NK, Delk JR, Hellstrom WJ, Cleves MA. Tramadol HCL has promise in on-demand use to treat premature ejaculation. J Sex Med, 2008; 5:188.

13. Choi HK, Jung GW, Moon KH, Xin ZC, Choi YD, Lee WH, et al. Clinical study of SS-cream in patients with lifelong premature ejaculation. Urology, 2000; 55:257.

14. Choi HK, Xin ZC, Choi YD, Lee WH, Mah SY, Kim DK. Safety and efficacy study with various doses of SS-cream in patients with premature ejaculation in a double-blind, randomized, placebo controlled clinical study. Int J Impot Res, 1999; 11:261. 